\title{
Les facteurs génétiques dans l'étiologie de la maladie d'Alzheimer
}

\section{Dominique Campion}

Alexis Brice

Didier Hannequin

Thierry Frebourg

Maria Martinez

Yves Agid

Françoise

Clerget-Darpoux

\section{Réseau Inserm \\ "Génétique \\ de la Maladie \\ d'Alzheimer "}

La maladie d'Alzheimer a un déterminisme très hétérogène. Dans les rares cas, à début précoce et à transmission autosomique dominante, trois gènes sont impliqués : le premier, localisé sur le chromosome 21 , code pour la protéine précurseur de l'amyloïde, le deuxième, sans doute le plus fréquemment en cause, est situé sur le chromosome 14 et code pour la préséniline 1 ; le troisième code pour la préséniline 2 , et est localisé sur le chromosome 1. L'existence d'un quatrième locus contenant un gène majeur est vraisemblable. A côté de ces gènes à transmission dominante, d'autres gènes sont des facteurs de risque pour la maladie; on a trouvé une association forte entre la maladie d'Alzheimer et l'allèle $\varepsilon 4$ du gène codant pour l'apolipoprotéine $E$. Il est encore trop tôt pour proposer un mécanisme pathogénique unique impliquant l'ensemble de ces gènes. Cependant, il est probable que l'action de plusieurs d'entre eux s'intègre dans le cadre de la cascade amyloïdergique, aboutissant à la constitution des plaques séniles et des lésions et symptômes associés.

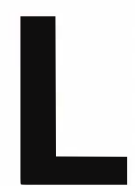

a maladie d'Alzheimer (MA) constitue un problème majeur de santé publique en raison de sa prévalence importante dans la population âgée et de la gravité du tableau clinique. Cette affection neurodégénérative dont il n'existe pas de traitement curatif se manifeste par des troubles de la mémoire et une altération des fonctions intellectuelles. L'évolution progressive conduit à une perte totale de l'autonomie et la plupart des patients ne peuvent être maintenus à leur domicile. La mort survient en général entre 5 et 10 ans après le début des symptômes. La maladie est caractérisée par la présence de dépôts de substance $\beta$-amyloïde au sein des plaques séniles et par la dégénérescence neurofibrillaire de certaines populations de neurones. La perte neuronale affecte préférentiellement certaines structures cérébrales telle l'hippocampe. L'incidence et la prévalence de cette 


\section{RÉFÉRENCES}

1. Rocca WA, Hofman A, Brayne C, Breteler MMB, Clarke M, Copeland JRM, Dartigues JF, Engedal K, Hagnell O, Heeren TJ, Jonker C, Lindesay J, Lobo A, Mann AH, Mölsä PK, Morgan K, O'Connor DW, Droux ADS, Sulkava R, Kay DWK, Amaducci L. Frequency and distribution of Alzheimer's disease in Europe: a collaborative study of 1980-1990 prevalence findings. Ann Neurol 1991; 30 : $381-90$.

2. Ritchie $\mathrm{K}$, Robine JM. La démence sénile en France: une estimation de la charge actuelle et projection des tendances futures. médecine/sciences $1994 ; 10: 680-6$.

3. Van Duijn CM, Clayton D, Chandra V. Familial aggregation of Alzheimer's disease and related disorders: a collaborative reanalysis of case-control studies. Int J Epidemiol 1991 ; 20 : S13.

4. McKhann G, Drachman D, Folstein M. Clinical diagnosis of Alzheimer's disease: report of the NINCDS-ADRDA work group under the auspices of department of health and human services task force on Alzheimer's disease. Neurology 1984 ; 34 : 939-44.

5. Mohs RC, Breitner JCS, Silverman JM, Davis KL. Alzheimer's disease: morbid risk among first degree relatives approximates $50 \%$ by 90 years of age. Arch Gen Psych 1987 ; 44: 405-8.

6. Campion D, Martinez M, Hannequin D, Brice A, Babron MC, Thomas-Anterion C, Michon A, Dubois B, Goas Y, Ledoze F, Pasquier F, Puel M, Zimmerman MA, Bellis M, Mallet J, Agid Y, Clerget-Darpoux F. Characteristics of familial aggregation in early-onset Alzheimer's disease: evidence of subgroups. Am J Med Genet 1995; 60 : $221-7$.

7. Farrer LA, O'Sullivan DM, Cupples A, Growdon JH, Myers RH. Assessment of genetic risk for Alzheimer's disease among first degree relatives. Ann Neurol 1989; 25: 485-93.

8. Van Duijn CM, Farrer LA, Cupples A, Hofman A. Genetic transmission of Alzheimer's disease among families in a Dutch population based study. J Med Genet 1993; 30 : $640-6$.

9. Martinez $\mathbf{M}$, Campion D, Babron MC, Hannequin D, Agid Y, Bellis A, Mallet J, Thomas-Anterion C, Clerget-Darpoux F. Segregation analysis of French Alzheimer pedigrees: rare dominant mutation(s) explains a minority of early-onset cases. $\mathrm{Am} \mathrm{J}$ affection augmentent en fonction de l'âge. Selon une étude collaborative européenne [1] le taux de prévalence est de $0,02 \%$ entre 30 et 60 ans, $0,3 \%$ entre 60 et 70 ans, $3,2 \%$ entre 70 et 80 ans et $10,8 \%$ après 80 ans. On estime qu'il y avait en 1994 248000 personnes atteintes de maladie d'Alzheimer en France et que ce chiffre atteindra 292000 cas en l'an 2000 du fait du vieillissement de la population [2]. De nombreuses études épidémiologiques de type castémoins ont cherché à identifier les facteurs de risque liés à cette maladie. Le facteur qui a été le plus communément retrouvé est l'existence d'une histoire familiale positive. Une méta-analyse récente [3] montre que le risque relatif (RR) pour les sujets ayant au moins un apparenté du premier degré atteint est de 3,5 avec un intervalle de confiance (IC) de $95 \%$ compris entre 2,6 et 4,6. Ce risque relatif est plus bas chez les patients n'ayant qu'un apparenté atteint $(2,6$, IC. 2,0-3,5) que chez les patients ayant au moins deux apparentés malades $(\mathrm{RR}=7,5, \mathrm{IC}=3,3-16,7)$. En présence de cette concentration familiale, il convient de rechercher les facteurs étiologiques susceptibles d'intervenir. Alors que l'implication de facteurs liés à l'environnem ent est toujours soumise à controverse, des résultats majeurs concernant le rôle des facteurs génétiques ont été obtenus ces dernières années. Les premières études quantifiant le risque morbide pour la MA chez les apparentés de premier degré de proposants estimaient ce risque à environ $50 \%$ au-delà de 80 ans (comparé à un risque de $10 \%$ pour les apparentés du premier degré de sujets témoins) [5]. Ce chiffre suggérait une transmission gé nétique autosomique dominante avec une pénétrance (probabilité d'être atteint si l'on est porteur du gé notype pathologique) complète dans la $9^{\mathrm{C}}$ décennie: pour tout patient, $50 \%$ des apparentés du premier degré seraient donc porteurs du génotype pathologique; toutefois, seule une fraction de ces individus développeraient réellement la maladie du fait de la mortalité intercurrente alors que les sujets sont encore dans la période à risque. La censure des données empêcherait d'observer les taux mendéliens sousjacents.

\section{Une étude collaborative française}

Depuis 1991 un réseau de recherche clinique composé de plusieurs unités de l'Inserm et du Cnrs associées à des centres cliniques a entrepris une étude familiale systématique des MA. Nous avons identifié un nombre important de proposants provenant d'admissions consécutives au sein de plusieurs services de neurologie hospitalo-universitaires. Tous ces sujets étaient atteints de MA probable selon les critères NINCDS-ADRDA [4]. A partir des proposants nous avons mené une enquête concernant les membres de la fratrie, les parents ainsi que les oncles/tantes paternels et maternels. L'analyse de ces familles a permis de mieux comprendre le déterminisme, très hétérogène, des MA.

\section{Pas de mode de transmission simple}

Nous avons d'abord testé l'hypothèse d'une transmission dominante à pénétrance complète sur un échantillon de 92 familles dont les proposants correspondaient tous à des cas précoces (âge de début $<60$ ans). Dans ces familles, nous disposions d'une très bonne information puisque nous avions pu examiner directement environ $80 \%$ des apparentés vivants. Pour les personnes décédées, le statut clinique a été établi en recoupant les informations auprès de plusieurs informants et en consultant les dossiers hospitaliers. Les risques morbides pour la MA chez les apparentés de premier degré atteignent seulement $35 \%$ à 90 ans et la valeur de $50 \%$ n'est pas comprise dans l'intervalle de confiance sur cette estimation [6]. Ces conclusions sont également celles d'autres études récentes [7,8] qui montrent que les risques morbides dans la $9^{e}$ décennie sont en réalité plus faibles que ceux initialement estimés.

\section{Une sous-entité mendélienne au sein des formes précoces}

Nous avons alors cherché à répondre à la question suivante: ce mode de 
transmission simple étant exclu, la concentration familiale observée dans les familles de ces 92 propoquer par un mode de transmission génétique homogène? Pour répondre à cette question nous avons utilisé l'analyse de ségrégation. Cette méthode permet de calculer la vraisemblance de différentes hypothèses de transmission du caractère étudié, pour l'échantillon familial recueilli. Cette analyse nous a permis de rejeter l'hypothèse d'une homogénéité génétique dans la transmission de la MA au sein de nos familles [9]. Cependant, nos résultats montrent que pour une minorité de cas à début précoce, la maladie se transmet bien selon un mode autosomique dominant avec une pénétrance complète à 60 ans (malades de type 1). Pour les autres cas (cas isolés ou cas familiaux de type 2) on ne peut pas mettre en évidence un déterminisme mendélien simple. La figure 1 montre la répartition de ces différents types de cas au sein des 92 familles. Dans c et échantillon provenant $d$ 'admissions hospitalières consécutives, les proposants de type 1 représentent environ $18 \%$ des personnes ayant débuté leur maladie avant 60 ans. Ce chiffre est plus sants peut-elle néanmoins s'expli-

faible que ceux habituellement rapportés dans la littérature mais il faut souligner que la plupart de ces estimations ont été réalisées à partir de séries totalement biaisées en faveur des formes familiales. La seule étude qui, à notre connaissance, a systématiquement échantillonné des patients à partir d'une population de référence donne une estimation encore plus basse que la nôtre (10\%) pour les formes autosomiques dominantes [10]. Comme la prévalence de l'ensemble de ces cas précoces est d'environ 8000 cas en France, on peut estimer que moins d'un millier de personnes sont concernées par les formes autosomiques dominantes de la MA.

\section{Le gène de l'APP sur le chromosome 21}

Ces dernières années de gros efforts ont été réalisés afin d'identifier les gènes responsables de la MA dans les familles de type 1. Les approches utilisées combinent les stratégies de clonage positionnel (avec cartographie du gène par étude de liaison génétique, puis identification de celui-ci dans la région ainsi délimitée) et de gènes candidats. L'étude

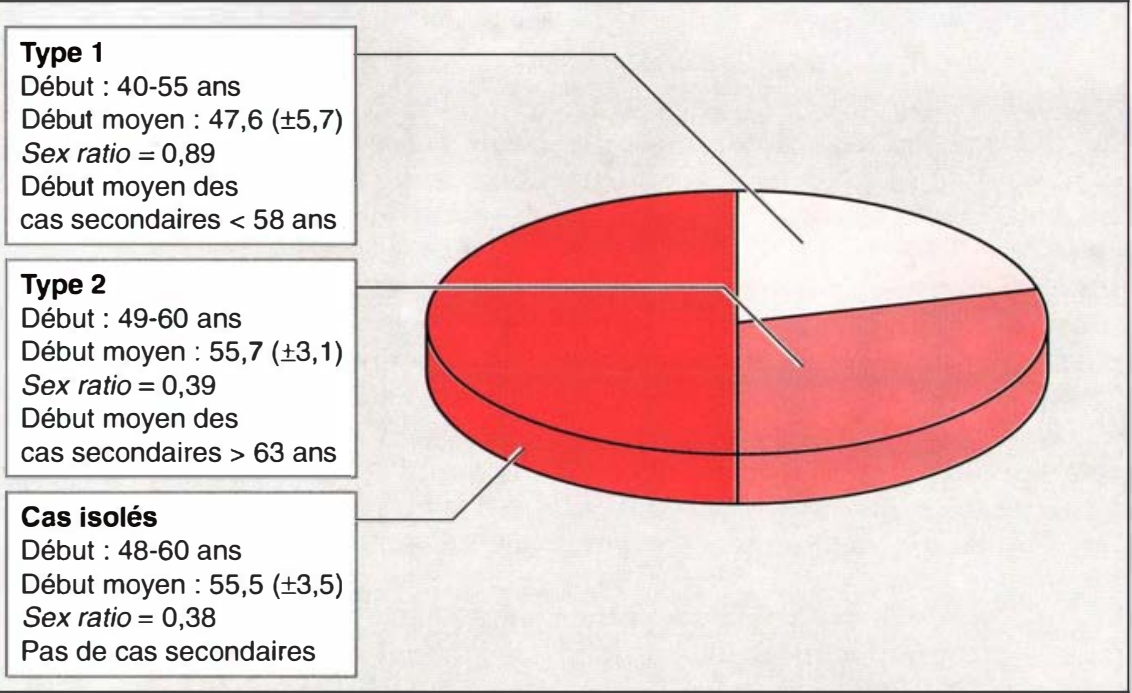

Figure 1. Répartition des cas isolés et des cas familiaux de types 1 et 2 dans un échantillon de 92 proposants présentant une MA ayant débuté avant 60 ans. Type 1 : cas familiaux à début précoce, à transmission autosomique dominante avec pénétrance complète à 60 ans ; type 2: autres cas familiaux. de StGeorges-Hyslop et al. en 1987 [11] fut la première à conclure à une liaison génétique avec des marqueurs de la région proximale du bras long du chromosome 21 dans quatre familles de MA précoce. Ce résultat s'avéra par la suite faux dans les familles considérées qui présentent, en réalité, toutes une mutation sur le gène de la préséniline 1 situé sur le chromosome 14 [12]. Cependant ce travail a eu le mérite d'engendrer un grand intérêt pour le chromosome 21 et plus particulièrement pour le gène du précurseur du peptide $\beta$ amyloïde (APP) situé en 2lq. Il était en effet assez naturel d'attribuer un rôle dans la physiopathologie de la maladie à ce gène puisque le principal constituant de la plaque sénile, le peptide $A \beta$, est un produit du catabolisme de l'APP. Plusieurs équipes ont donc recherché des altérations du gène $A P P$ qui code, par épissage alternatif, pour différents transcripts dont le plus long correspond à une protéine de 770 acides aminés [13]. En 1991, une première mutation remplaçant une valine par une isoleucine au codon 717 a été mise en évidence chez les patients d'une famille anglaise [14]. Simultanément d'autres mutations de l'APP, dont certaines concernaient également le codon 717, ont été décrites [15-17]. Ces mutations se situent aux 2 extrémités de la séquence codant pour le peptide $\mathrm{A} \beta$ (voir figure 2) c'est-à-dire à proximité des sites de clivage du peptide sur le précurseur. En 1993, nous avons décrit une mutation substituant une thréonine à une valine au codon 713 de l'APP chez une patiente présentant une forme précoce de MA [18]. Cette mutation se situe au site de clivage qui produit la forme la plus longue ( 42 acides aminés) du peptide $A \beta$. Si elle est pathogène, sa pénétrance, contrairement aux autres mutations de l'APP, est incomplète puisque plusieurs apparentés âgés sont porteurs asymptomatiques. Nous avons également documenté des mutations 717 Val $\rightarrow$ Ile dans trois autres familles. Actuellement, moins d'une vingtaine de familles liées à des mutations APP ont été observées dans le monde. Au regard de ce faible nombre il est rapidement apparu qu'il existait une hétérogénéité génétique au sein même des familles de type 1 . 


\section{RÉFÉRENCES}

10. Van Duijn CM, Knijff $P$, Cruts $M$, Wehnert A, Havekes LM, Hofman A, Van Broeckhoven C. Apolipoprotein E4 allele in a population-based study of early onset $\mathrm{Alz}$ heimer's disease. Nature Genet 1994; 7: 748.

11. St George-Hyslop PH, Tanzi RE, Polinsky RJ, Haines JL, Nee L, Watkins PC, Myers RH, Feldman RG, Pollen D, Drachman D Growdon J, Bruni A, Foncin JF, Salmon D, Frommelt P, Amaducci L, Sorbi S, Piacentini S, Stewart GD, Hobbs WJ, Conneally M Gusella JF. The genetic defect causing FAD maps on chromosome 21. Science $1987 ; 235$ $885-9$.

12. Sherrington $R$, Rogaev EI, Liang $Y$, Rogaeva EA, Levesque G, Ikeda M, Chi $\mathrm{H}$, Lin C, Holman K, Tsuda T, Mar L, Foncin JF, Bruni AC, Montesi MP, Sorbi S, Rainero I, Pinessi L, Nee L, Chumakov, Pollen D, Brookes A, Sanseau P, Polinsky RI, Wasco W, Da Silva HAR, Haines JL, Pericak-Vance MA, Tanzi RE, Roses AD, Fraser PE, Rommens JM, St George-Hyslop PH. Cloning of a gene bearing missense mutations in earlyonset familial Alzheimer's disease. Nature $1995 ; 375$ : $754-60$

13. Octave J, Macq A, Philippe B. Le précurseur du peptide amyloïde de la maladie d'Alzheimer. médecine/sciences $1995 ; 11$ : 1251-9.

14. Goate A, Chartier-Harlin MC, Mullan M, Brown J, Crawford F, Fidani L, Giuffra L, Haynes A, Irving N, James L, Mant R, Newton P, Rooke K, Roques P, Talbot C, Pericak-Vances M, Roses A, Williamsom R, Rossor M, Owen M, Hardy J. Segregation of a missense mutation in the amyloid precursor gene with familial Alzheimer's disease. $\mathrm{Na}$ ture 1991 ; 349 : 7046.

15. Chartier-Harlin MC, Crawford F, Houlden $\mathrm{H}$, Warren A, Hughes D, Fidani L, Goate A, Rossor M, Roques P, Hardy J, Mullan M. Early onset Alzheimer's disease caused by mutations at codon 717 of the $\beta$ amyloid precursor protein gene. Nature 1991 ; 353 : 8445 .

16. Mullan M. A pathogenic mutation for probable Alzheimer's disease in the APP gene at the $\mathrm{N}$-terminus of $\beta$-amyloid. Nature Genet 1992; 1 : 345-7.

17. Murrel J, Farlow M, Ghetti B, Benson $\mathrm{MD}$. A mutation in the amyloid precursor protein associated with hereditary Alzheimer's disease. Science 1991 ; 254 : $97-9$.

18. Carter DA, Desmarais E, Bellis M, Campion D, Brice A, Agid Y, Jaillard A, Clerget-
Darpoux $F$. More missense in amyloid gene. Nature Genet 1992; 2: 255.

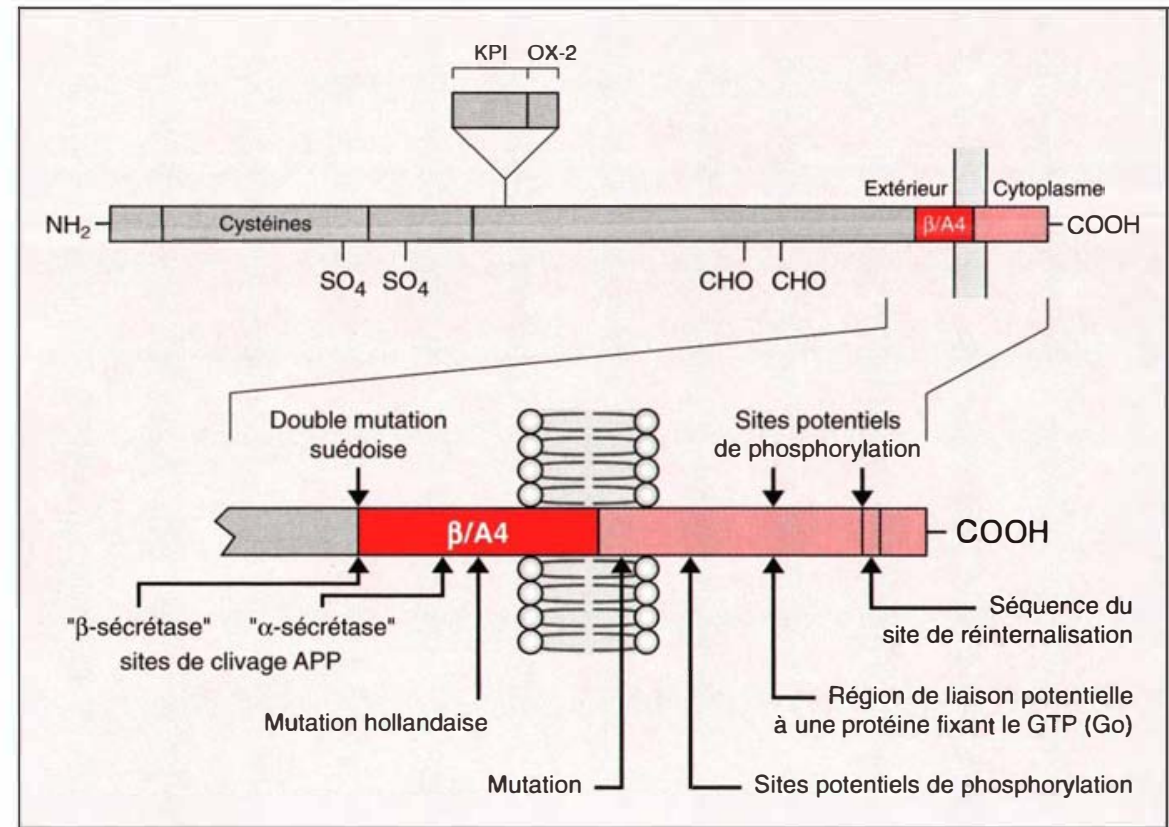

Figure 2. Protéine APP. A : représentation de l'ensemble de la protéine précurseur de la protéine amyloïde. Le gène APP code, par épissage alternatif, pour différents transcrits dont le plus long comporte 770 acides aminés. La protéine a un important domaine extracellulaire amino-terminal qui contient une région riche en cystéines, des sites de glycosylation (CHO), des régions inhibitrices de protéases à sérine (KPI) et un domaine analogue à un antigène lymphocytaire (OX2). Le domaine à l'origine du peptide $A_{4}-\beta$, indiqué en rouge, est en partie extracellulaire et en partie transmembranaire. $B$ : agrandissement du domaine carboxyterminal. Le site de clivage par l' $\alpha$-sécrétase se situe au milieu du peptide $\beta$. A l'extrémité aminoterminale du peptide $\beta / A_{4}$ est localisé le site de clivage par la $\beta$-sécrétase. Les mutations situées sur les exons 16 et 17 du gène correspondent aux sites de clivage du peptide $A-\beta$ sur son précurseur. Les mutations "suédoise" et "hollandaise" interfèrent avec le catabolisme de I'APP et augmentent ainsi la production de peptide $A \beta$.

\section{Un second gène sur le chromosome 14}

Au cours de l'année 1992, une seconde liaison avec des marqueurs du chromosome 14 a été mise en evidence dans des familles de type $1 \mathrm{~lm} / \mathrm{s}$ $n^{\circ} 10$, vol. 8, p. 1115) [19]. Ce résultat a rapidement été confirmé par plusieurs équipes [20, 21]. Pour notre part, nous avons pu montrer que, dans plusieurs de nos familles de type 1 , le gène pathologique se situait à cette localisation chromosomique. En particulier, nous avons caractérisé une famille très étendue comportant 35 sujets atteints (figure 3 ) [22]. La généalogie a été reconstituée à partir de plusieurs proposants de type 1 portant tous un allèle rare pour le marqueur D14S43 situé à proximité du locus pathologique [23]. Un étude généalogique nous a ensuite permis de relier ces proposants à deux ancêtres communs nés respectivement en 1804 et 1808. Dans cette famille l'âge de début de la maladie est compris entre 39 et 52 ans (moyenne $46 \pm 3,5$ ans) et la transmission est autosomique dominante.

\section{Les présénilines 1 et 2}

Le gène localisé sur le chromosome 14 a finalement été cloné en juin $1995\left(\mathrm{~m} / \mathrm{s} n^{\circ} 9\right.$, vol. 11, p. 1354) [12]. Il code pour une protéine de 467 acides aminés nommée préséniline 1 . De nombreuses mutations faux sens (c'est-à-dire substituant un acide a miné par un autre sur la séquence de la protéine) ont été décrites sur ce gène. Dès que le gène de préséniline 1 a été connu il a été possible de cribler des banques d'ADNc à la recherche de gènes homologues. Cela a permis d'identifier un troisiè- 


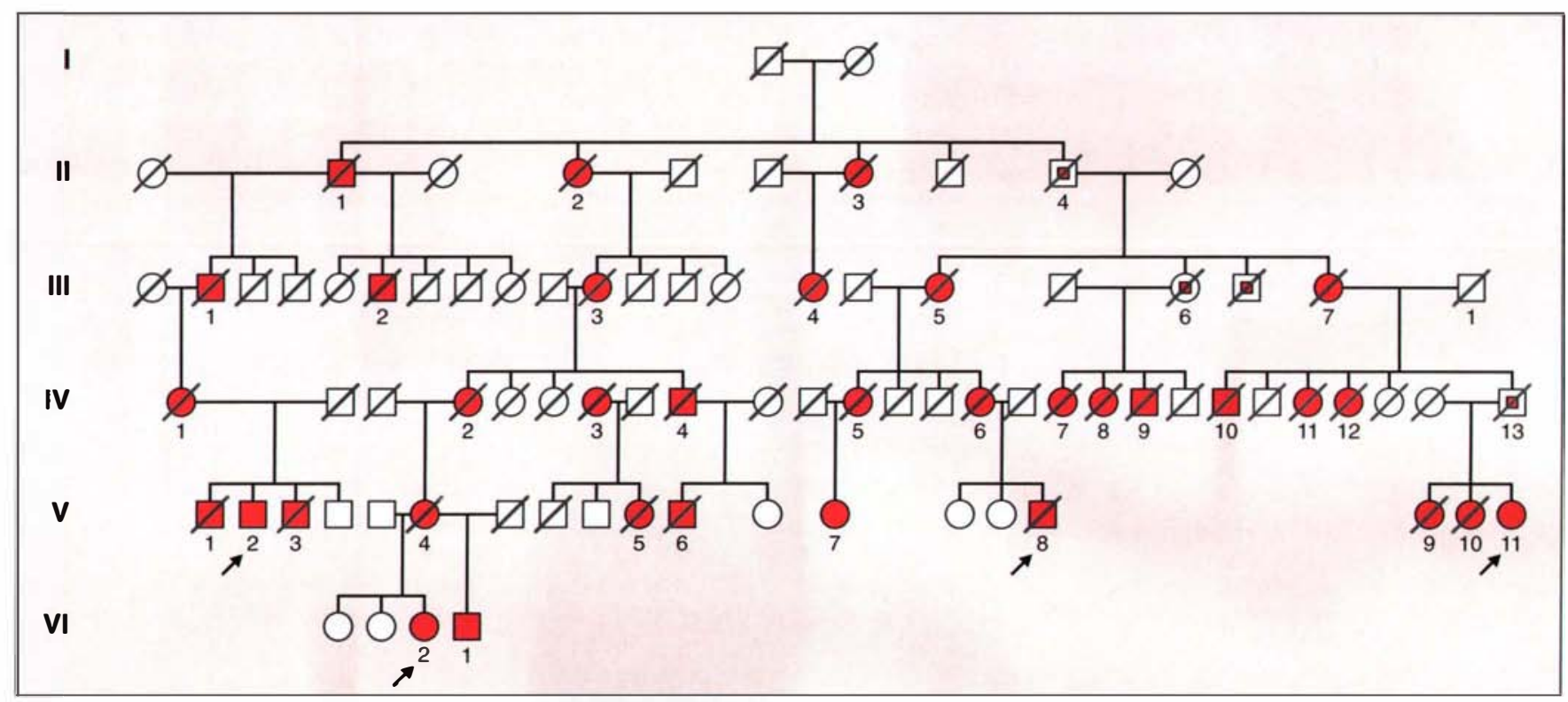

Figure 3. Mutation de la préséniline 1 dans une famille comptant 35 sujets atteints. Age de début moyen, $46 \pm$ 3,5 ans. Les ancêtres sont nés en 1804 et 1808. La transmission est de toute évidence autosomique dominante. Les flèches indiquent les proposants. Les carrés noirs indiquent un transmetteur obligatoire décédé jeune, en rouge, les sujets atteints.

me gène impliqué dans la sous-entité mendélienne précoce [24]. Ce gène, situé sur le chromosome 1 , code pour la préséniline 2, une protéine de 475 acides aminés qui présente $80 \%$ de similitude de séquence avec la préséniline 1 . Une mutation de ce gène est responsable des cas de MA présents dans un isolat ethnique particulier, celui des "Allemands de la Volga $"\left(m / s n^{\circ} 11\right.$, vol. 11, p. 1610). Mis à part ce groupe dans lequel il existe un effet fondateur (une même mutation est présente dans toutes les familles), une seule autre famille italienne liée à une mutation de la préséniline 2 a pour l'instant été décrite.

\section{Qu'en est-il dans les familles françaises?}

A l'étape actuelle, nous avons identifié un total de 16 familles de type 1 . Le Tableau I décrit les caractéristiques de ces 16 familles ainsi que le détail des mutations qui y ségrègent. Dans 3 familles la mutation retouvée est une mutation de l'APP qui entraîne le remplacement d'une valine par une isoleucine au codon 717. Dans 8 autres familles, il existe des mutations faux sens sur le gène de la préséniline 1 [25]. Ce résultat confirme que la préséniline 1 constitue le gène majeur responsable de ces formes. Aucune mutation de la préséniline 2 n'a été retrouvée dans ces familles. Enfin, il existe des familles négatives pour les trois gènes connus, ce qui suggère l'existence d'au moins un quatrième locus intervenant dans ces formes précoces à transmission dominante.

\section{Corrélations génotype-phénotype}

Existe-t-il une différence de présentation clinique entre les MA dues à des mutations de l'APP et cell es dues à la préséniline 1 ? Il es t actuel lement difficile de répondre à cette question avec précision. L’âge de début est

\begin{tabular}{|c|c|c|c|c|}
\hline \multicolumn{5}{|c|}{ Tableau I } \\
\hline Famille & $\begin{array}{l}\text { Malades } \\
\text { (nombre) }\end{array}$ & $\begin{array}{l}\text { Âge de début } \\
\text { (extrêmes) }\end{array}$ & Gène & $\begin{array}{l}\text { Mutation } \\
\text { (codon) }\end{array}$ \\
\hline FAD RO1 & 35 & $39-52$ & préséniline 1 & Leu $^{392} \rightarrow$ Val \\
\hline ALZ 042 & 3 & 45 & préséniline 1 & $\mathrm{Ala}^{231} \rightarrow \mathrm{Thr}$ \\
\hline ALZ 025 & 2 & $35-37$ & préséniline 1 & $\mathrm{Tyr}^{115} \rightarrow \mathrm{His}$ \\
\hline ALZ 028 & 4 & $45-49$ & ND & \\
\hline ALZ 506 & 4 & $46-52$ & ND & \\
\hline ROU 011 & 14 & $44-60$ & préséniline 1 & $\mathrm{Cys}^{410} \rightarrow \mathrm{Tyr}$ \\
\hline SAL 508 & 3 & $53-58$ & préséniline 1 & $\mathrm{Val}^{82} \rightarrow$ Leu \\
\hline SAL 511 & 6 & $45-58$ & préséniline 1 & Pro $^{264} \rightarrow$ Leu \\
\hline ALZ 013 & 9 & $45-56$ & ND & \\
\hline ALZ 009 & 5 & $50-57$ & $N D$ & \\
\hline ALZ 036 & 3 & $52-61$ & ND & \\
\hline CAE 010 & 2 & $48-50$ & préséniline 1 & $\mathrm{Met}^{139} \rightarrow \mathrm{Thr}$ \\
\hline SAL 005 & 2 & $42-47$ & préséniline 1 & $\mathrm{His}^{163} \rightarrow$ Arg \\
\hline FAD RO3 & 6 & $50-60$ & $A P P$ & $\mathrm{Val}^{717} \rightarrow \mathrm{Ile}$ \\
\hline FAD RO4 & 5 & $53-55$ & $A P P$ & $\mathrm{Val}^{717} \rightarrow \mathrm{Ile}$ \\
\hline FAD SAL 1 & 9 & $38-51$ & $A P P$ & $\mathrm{Val}^{717} \rightarrow \mathrm{Ile}$ \\
\hline
\end{tabular}

* ND: non déterminé. 


\section{RÉFÉRENCES}

19. Schellenberg GD, Bird TD, Wijsman EM, Orr HT, Anderson L, Nemens E, White JA, Bonnycastle L, Weber JL, Alonso E, Potter $\mathrm{H}$, Heston LL, Martin GM. Genetic linkage evidence for a familial Alzheimer's disease locus on chromosome 14. Science 1992 ; 258: 668-71.

20. Van Broeckhoven $C$, Backhovens $H$, Cruts M, De Winter G, Bruyland M, Cras P, Martin JJ. Mapping of a gene predisposing to early-onset Alzheimer's disease to chromosome 14q24.3. Nature Genet 1992; 2 : 335-9.

21. St George-Hyslop P, Haines J, Rogaev E, Mortilla M, Vaula G, Pericak-Vance M, Foncin JF, Montesi M, Bruni A, Sorbi S, Rainero I, Pinessi L, Pollen D, Polinsky R, Nee L, Kennedy J, Marcciardi F, Rogaeva E, Liang Y, Alexandrova N, Lukiw W, Schlumpf $\mathrm{K}$, Tanzi R, Tsuda T, Farrer L, Cantu JM, Duara R, Amaducci L, Bergamini L, Gusella J, Roses A, McLachlan C. Genetic evidence for a novel familial Alzheimer's disease locus on chromosome 14. Nature Genet 1992; 2: $330-4$.

22. Campion D, Brice A, Hannequin D, Tardieu S, Dubois B , Calenda A, Bellis M, Martinez M, Agid Y, Clerget-Darpoux F. A large pedigree with early-onset Alzheimer's disease : clinical, neuropathologic, and genetic characterization. Neurology, 1995 45: 80-5.

23. Brice A, Tardieu S, Campion D, Le Guern E, Martinez M, Carpentier A, Penet C, Dubois B, Bellis M, Mallet J, Hannequin D, Clerget-Darpoux F, Agid Y. Allelic association at the D14S43 locus in early onset Alzheimer's disease. Am I Med Genet 1995; 60: $91-3$.

24. Levy-Lahad E, Wasco W, Poorkaj P, Romano DM, Oshima I, Pettingell WH, Yu CY, Jondro PD, Schmidt SD, Wang K, Crowley AC, Fu YH, Guenette SY, Galas D, Nemens E, Wijsman EM, Bird TD, Schellenberg GD, Tanzi RE. Candidate gene for the chromosome 1 familial Alzheimer's disease locus. Science $1995 ; 269$ : 973-7.

25. Campion D, Flaman JM, Brice A, Hannequin D, Dubois B, Martin C, Moreau V, Charbonnier $\mathrm{F}$, Didierjean $\mathrm{O}$, Tardieu $\mathrm{S}$, Penet C, Puel M, Pasquier F, Ledoze F, Bellis $G$, Calenda A, Heilig R, Martinez M, Mallet J, Bellis M, Clerget-Darpoux F, Agid Y, Frebourg Th. Mutations of presenilin 1 gene in families with early-onset Alzheimer's disease. Hum Mol Genet 1995 ; 4 : 23737.

26. Hannequin D, Campion D, Tardieu S, Auzou P, Martinez M, Dubois B, ClergetDarpoux F, Agid Y, Brice A. Phénotype des formes familiales précoces de maladie d'Alzheimer liées au chromosome 14. Caractéristiques cliniques et neuropsychologiques d'une grande famille. Rev Neurol $1995 ; 151: 682-90$ plus précoce dans les familles liées au chromosome 14. La durée de l'évolution est identique. Des signes neurologiques associés (crises d'épilepsie, syndrome extrapyramidal, myoclonies) sont rencontrés avec une fréquence élevée dans les deux formes [26].

\section{L'APO E4 facteur de risque}

Pour intéressantes qu'elles soient, ces découvertes ne concernent qu'un nombre très restreint de cas. Il était donc nécessaire d'étudier le déterminisme des autres cas de MA. Dès 1987, le groupe de G. Schellenberg avait attiré l'attention sur l'existence d'une association entre la MA et un allèle du gène $A P O C I I$ situé sur le chromosome 19 [27]. En 1992 cette association a été retrouvée au niveau du locus $A P O E$, très proche du précédent [28]. Dans la population, il existe 3 variants du gène $A P O E$ appelés allèles $\varepsilon 2, \varepsilon 3$ et $\varepsilon 4$. Ces variants diffèrent par des substitutions concernant deux acides aminés sur la protéine mûre. L'allèle le plus représenté est l'allèle $\varepsilon 3$ avec une fréquence d'environ $80 \%$, la fréquence de l'allèle $\varepsilon 4$ étant de $15 \%$ et celle de l'allèle $\varepsilon 2$ de $5 \%$. L'allèle $\varepsilon 4$ est connu pour représenter un facteur de risque cardiovasculaire, ce qui explique sa moindre fréquence chez les sujets de sexe masculin après 50 ans $\left(\mathrm{m} / \mathrm{s} n^{\circ} 11\right.$, vol. 10, $p$. 1179) [29]. Initialement, il a été montré que la fréquence de l'allèle $\varepsilon 4$ était significativement plus élevée chez les patients attein ts de M A à âge de début tardif que chez des individus normaux appariés pour l'âge $\left(\mathrm{m} / \mathrm{s} n^{\circ}\right.$ 10, vol. 9, p. 1142; $n^{\circ} 4$, vol. $10, p .470)$. Cette observation a été répliquée [30] puis étendue aux formes à début préc oce [10, 31, 32]. Il existe un effet de dosage génique, le risque relatif étant plus important pour les individus dont le génotype est $\varepsilon 4 / \varepsilon 4$ [33]. Cependant il faut souligner que la présence de l'allè le $\varepsilon 4$ n'est ni indispensable (des individus non $\varepsilon 4$ peuvent être atteints) ni suffisante (des individus $\varepsilon 4$ peuvent ne pas être atteints même à un âge très avancé) pour déclencher la MA. L'APOE $\varepsilon 4$ est un facteur de risque génétique dont le déterminisme est différent de celui des mutations dominantes précédemment évoquées. Enfin, il a été suggéré qu'un autre allèle du même gène (APOE $\varepsilon 2)$ jouerait, quant à lui, un rôle protecteur vis-à-vis de la $\mathrm{MA}\left(\mathrm{m} / \mathrm{s} n^{\circ} 8 / 9\right.$, vol. 10, p. 920) [34].

\section{Quantifier les risques liés aux génotypes APOE}

Dans une étude concernant 427 patients et 1130 témoins [35] nous avons cherché à quantifier les risques relatifs associés à la présence de l'allèle $\varepsilon 4$ en fonction de l'âge de début de la maladie et du sexe. Les malades correspondent à des admissions consécutives dans des services hospitaliers alors que les témoins proviennent d'individus sélectionnés au hasard sur des listes de résidents de maisons de retraites. Tous les témoins ont fait l'objet d'une évaluation neuro-psychologique. Le Tableau II montre l'augmentation de fréquence de l'allèle $\varepsilon 4$ chez les malades comparés aux témoins. Le risque conféré par l'allèle $\varepsilon 4$ est maximal dans la tranche 60-70 ans et n'est pas significativement différent pour les hommes et les femmes. Nous avons ensuite classé les patients en fonction de le ur histoire familiale. En étudiant un sous-groupe de patients ayant tous un début précoce ( $<60$ ans) nous avons ainsi pu montrer que la présence de l'allè le $\varepsilon 4$ chez un malade augmente le risque d'être atteint pour ses apparentés (Tableau III). Toutefois, comme on pouvait s'y attendre, pour les proposants des familles de type 1 , porteurs d'une mutation dominante, il n'existe pas d'association avec l'allèle $\varepsilon 4$ [32]. Beaucoup de travail reste encore à effectuer avant que les courbes de risque associées aux différents gén otypes APOE en fonction de l'âge et du sexe soient connues avec précision.

\section{Un nouvel éclairage sur la physiopathologie}

Comment intégrer toutes ces données en un modèle pathogénique cohérent ? Pour reconstituer la physiopathologie des MA nous ne disposions, jusqu'à ces dernières années, que d'un modèle indirect, celui de la trisomie 21. De nombreux trisomiques présentent en effet les stigmates anatomo-pathologiques de la 
Tableau ॥

FRÉOUENCE DE L'ALLÈLE APOE-£4 EN FONCTION DE L'ÂGE ET DU SEXE CHEZ 417 PATIENTS ET 1130 TÉMOINS

\begin{tabular}{|c|c|c|c|c|c|c|c|c|c|c|c|c|}
\hline \multicolumn{2}{|c|}{ Classe d'âge } & \multicolumn{2}{|c|}{$<60$ ans } & \multicolumn{2}{|c|}{$60-69$ ans } & \multicolumn{2}{|c|}{$70-79$ ans } & \multicolumn{2}{|c|}{$>80$ ans } & \multicolumn{2}{|c|}{ Total } & \multirow[t]{2}{*}{ Total } \\
\hline Sexe & & Hommes & Femmes & Homm & Femmes & Homm & Femmes & Homm & Femmes & Homme & Femmes & \\
\hline Cas & n & 52 & 82 & 38 & 84 & 35 & 75 & 9 & 42 & 134 & 283 & 417 \\
\hline $\begin{array}{l}f(4) \\
\text { Témoins }\end{array}$ & $\begin{array}{c}\% \\
\mathrm{n}\end{array}$ & $\begin{array}{l}27,9 \\
40\end{array}$ & $\begin{array}{l}30,5 \\
47\end{array}$ & $\begin{array}{l}32,9 \\
93\end{array}$ & $\begin{array}{l}50,0 \\
81\end{array}$ & $\begin{array}{l}35,7 \\
85\end{array}$ & $\begin{array}{l}32,7 \\
153\end{array}$ & $\begin{array}{l}27,8 \\
114\end{array}$ & $\begin{array}{l}23,8 \\
517\end{array}$ & $\begin{array}{l}31,3 \\
332\end{array}$ & $\begin{array}{l}35,9 \\
798\end{array}$ & 1130 \\
\hline$f(4)$ & $\%$ & 15,0 & 19,1 & 8,6 & 22,2 & 10,6 & 18,3 & 13,6 & 18,0 & 11,6 & 18,5 & 16,5 \\
\hline
\end{tabular}

$F$ (4): fréquence de l'allèle $\varepsilon 4$.

\section{RÉFÉRENCES}

27. Schellenberg GD, Deeb SS, Boehnke M, Bryant EM, Martin GM, Lampe TH, Bird TD. Association of an apolipoprote in CII allele with familial dementia of the Alzheimer type.J Neurogenetics 1987; 4: 97-108.

28. Saunders AM, Strittmatter WJ, Schmechel W, St Georges-Hyslop P, Pericak-Vance MA, Roses AD. Association of apolipoprotein allele $\mathrm{E} 4$ with late-onset Alzheimer's disease. Neurology 1993; 43: 1467-72.

29. Cummings AM, Robertson FW. Polymorphism at the apolipoprotein E locus in relation to risk of coronary disease. Clin Cienet $1984 ; 25: 310-3$.

30. Brousseau T, Legrain S, Berr C, Gourlet $\mathrm{V}$, Vidal O, Amouyel P. Confirmation of the $\varepsilon 4$ allele of the apolipoprotein $\mathrm{E}$ gene as a risk factor for late-onset Alzheimer's disease. Neurology 1994; 44: 342-4.

31. Chartier-Harlin MC, Parfitt M, Legrain S, Perez-Tur J, Brousseau T, Evans A, Berr C, Vidal O, Roques P, Gourlet V, Fruchart JC, Delacourte A, Rossor M, Amouyal P. Apolipoprotein E, $\varepsilon 4$ allele as a major risk factor for sporadic early and late-onset forms of Alzheimer's disease : analysis of the $19 \mathrm{q} 13.2$ chromosomal region. Hum Mol (jenet $1994 ; 3: 569-74$.

32. Pérez-Tur J, Campion D, Martinez M, Brice A, Tardieu S, Hannequin D, Agid Y, Delacourte A, Clerget-Darpoux F, Chartier Harlin MC. Evidence for APOE $\varepsilon 4$ association in early-onset Alzheimer's disease patients with late onset relatives. Am J Med Genet $1995 ; 60: 550-3$.

33. Corder EH, Saunders AM, Strittmatter WJ, Schmechel DE, Gaskell PC, Small GW, Roses $\mathrm{AD}$, Haines JL, Pericak-Vance MA. Gene dose of apolipoprotein E type 4 allele and the risk of Alzheimer's disease in late on set families. Science 1993; 261 : 921-3.

34. Corder EH, Saunders AM, Risch NJ, Strittmatter WJ, Schmechel DE, Gaskell PC, Rimmler JB, Locke PA, Conneally PM, Schmader KE, Small GW, Roses AD, Haines JL, Pericak-Vance MA. Protective effect of apolipoprote in E type 2 allele for late onset Alzheimer disease. Nature (ienet 1994; 7: $180-4$.

$\mathrm{m} / \mathrm{s} n^{\circ} 6-7$, vel. 12 , juin-juillet 96
MA après l'âge de 40 ans [36]. Dans ce cas, il est généralem ent admis que le primum movens de l'affection est la surexpression du gène $\mathrm{APP}$, conduisant à une accumulation de substance amyloïde (peptide $A \beta$ ), laquelle induit par un mécanisme encore inconnu la dégénérescence neurofibrillaire et la mort de certaines populations neuronales [37].

Les conséquences des différentes mutations de l'APP précédemment décrites ont été étudiées in vitro. La double mutation située aux codons 670/671 (mutation suédoise) modifie le métabolisme normal de l'APP et conduit à une augmentation de la production du peptide $A \beta$ dans des cellul es transfectées en culture [38] et dans les fibroblastes de patients porteurs de cette mutation. Les mutations du codon 717 modifient également le catabolisme de l'APP et favorisent la production des dérivés les plus longs (42 acides aminés au lieu de 39 acides aminés) du peptide $\mathrm{A} \beta$ [39]. Ces dérivés ont une tendance plus marquée à l'agrégation en fragments insolubles caractéristiques des plaques séniles. La caractérisa-

\section{Tableau III}

GÉNOTYPES APO E CHEZ 119 PATIENTS CLASSÉS SELON LEUR HISTOIRE FAMILIALE ET 109 TÉMOINS. LES PROPOSANTS ONT TOUS UN ÂGE DE DÉBUT < 60 ANS

\begin{tabular}{|c|c|c|c|c|c|}
\hline \multicolumn{6}{|c|}{$\begin{array}{l}\text { GÉNOTYPES APO E CHEZ } 119 \text { PATIENTS CLASSÉS } \\
\text { SELON LEUR HISTOIRE FAMILIALE ET } 109 \text { TÉMOINS. } \\
\text { LES PROPOSANTS ONT TOUS UN ÂGE DE DÉBUT < } 60 \text { ANS }\end{array}$} \\
\hline Proposants & $\mathbf{n}$ & $\varepsilon 2,3 / \varepsilon 2,3$ & $\varepsilon 2,3 / \varepsilon 4$ & $\varepsilon 4 / \varepsilon 4$ & $f(\varepsilon 4)$ \\
\hline Type 1 & 18 & 12 & 5 & 1 & $19 \%$ \\
\hline Type 2 & 38 & 13 & 16 & 9 & $45 \%$ \\
\hline Isolés & 63 & 40 & 21 & 2 & $20 \%$ \\
\hline Total patients & 119 & 65 & 42 & 12 & $28 \%$ \\
\hline Témoins & 109 & 78 & 28 & 3 & $16 \%$ \\
\hline
\end{tabular}

Les proposants de type 1 correspondent aux familles dans lesquelles ségrège un gène dominant.

Les autres cas familiaux sont de type 2. $\{\varepsilon 4)$ = fréquence de l'allèle $\varepsilon 4$. tion des mutations de l'APP par le groupe de J. Hardy a donc correspondu à une avancée conceptuelle très importante en confirmant le rôle central joué par les dépôts de substance amyloïde dans la physiopathologie de la MA [13].

Les données relatives aux présénilines 1 et 2 sont beaucoup plus pauvres. Ces molécules présentent une homologie de séquence avec proteines se trouvant chez le nématode Caenorhabditis elegans. La première de ces protéines (SP4) joue un rôle dans la stabilisation des vésicules issues de l'appareil de Golgi. Comme la maturation de l'APP a lieu dans ce type de vésicules, il a été suggéré que les mutations des présénilines interféreraient in fine avec la production de peptide $A \beta$. De fait, dans des cellules humaines, les présénocytochimie au niveau du réticulum endoplasmique et de l'appareil de Golgi [40]. De plus, il a été rapporté que les fibroblastes de sujets présentant une mutation de la PS 1 sécrètent préférentiellement les dérivés les plus longs du peptide $A \beta$ [41]. Selon une 


\section{RÉFÉRENCES}

35. Bickeböller H, Campion D, Brice A Amouyel $\mathrm{Ph}$, Hannequin D, Didierjean $\mathrm{O}$, Penet C, Martin C, Perez-Tur J, Michon A, Dubois B, Ledoze F, Thomas-Anterion C Pasquier F, Puel M, Demonet JF, Moreaud $\mathrm{O}$, Babron MC, Meulien D, Guez D, Chartier-Harlin MC, Frebourg T, Agid Y, Martinez $\mathrm{M}$, Clerget-Darpoux F. APOE and $\mathrm{Alz}$ heimer's disease: age, sex and genotype specific relative risks and incidence rates. Proc Natl Acad Sci USA 1993; 90 : 9649-53.

36. Sinet P, Nicolle A, Ceballos I, Delabar I. Trisomie 21 et maladie d'Alzheimer. médecine/sciences $1987 ; 3: 256-62$.

37. Rumble B, Retallack R, Hilbich C, et al Amyloidd A4 protein and its precursor in Down's syndrome and Alzheimer's disease. N Engl J Med 1989; 320 : 1146-2.

38. Citron M, Oltersdorf T, Haass C, McConlogue L, Hung AY, Seubert P, Vigo-Pelfrey C, Lieberburg I, Selkoe DJ. Mutation of the amyloid precursor protein in familial Alzheimer's disease increases $\beta$-protein production. Nature 1992; 360:672-4.

39. Suzuki N, Tobun TC, Cai XD, Odaka A Otvos L, Eckman C, Golde TE, Younkin SG. An increased percentage of long amyloid $\beta$ protein secreted by familial amyloid $\beta$ protein precursor mutants. Science 1994; 264 : $1336-40$.

40. Kovacs DM, Fausett HJ, Page KJ, Kim TW, Moir D, Merriam DE, Hollister RD, Hallmark OG, Mancini R, Felsenstein KM, Hyman BT, Tanzi RE, Wasco W. Alzheimer associated presenilins 1 and 2: neuronal expression in brain and localization to intracellular membranes in mammalian cells. Nature Med 1996; 2 : 2249.

41. Scheuner D. Fibroblasts from carriers of familial AD linked to chromosome 14 show increased A $\beta$ production. Soc Neurosci Abstr $1995 ; 21: 1500$.

42. Dewji NN, Singer SJ. Genetic clues to Alzheimer's disease. Science 1996; 271 : 159-60.

43. Naslund J, Thyberg J, Tjernberg LO, Wernstedt C, Karlstrom AR, Bogdanovic N Gandy SE, Lannfelt L, Terenius L, Nordstedt $C$. Characterization of stable complexes involving apolipoprotein $E$ and the amyloid $\beta$ peptide in Alzheimer's disease brain. Neuron $1995 ; 15$ : 219-8.

44. Schmechel DE, Saunders AM, Strittmatter W, Crain BJ, Hulette CM, Joo SH, Pericak-Vance MA, Goldgaber D, Roses AD. Increased amyloid $\beta$-peptide deposition in cerebral cortex as a consequence of apolipoprotein E genotype in late-onset. Proc autre hypothèse fondée sur l'analogie de séquence entre la PS1 et Sell2, une autre protéine de nématode, la PS1 et l'APP pourraient interagir au niveau des membranes de cellules voisines. Il en résulterait l'internalisation de vésicules contenant ces complexes et la protéolyse de l'APP [42]. Ces données encore préliminaires sont compatibles avec l'intégration du gène PS1 dans le cadre de la cascade amyloïdergique.

\section{Le rôle de l'ApoE}

Concernant le rôle de l'allèle $\varepsilon 4 \mathrm{du}$ gène $A P O E$ dans la physiopathologie de la MA, notons tout d'abord qu'il existe actuellement un consensus pour admettre que c'est bien l'allèle E4 lui-même (et non une autre mutation en déséquilibre de liaison avec $\varepsilon 4)$ qui constitue le facteur de risque pour la MA. Plusieurs hypothèses ont été avancées pour rendre compte du rôle de l'isoforme E4. La plus documentée est basée sur la détection, par immunoh istochimie, de molécules d'APOE au sein des plaques séniles. Il a été montré in vivo et in vitro que ces molécules forment des complexes stables avec le peptide $A \beta[43]$. Elles se comporteraient comme des chaperons moléculaires intervenant dans la conformation du peptide $A \beta$ en une forme insoluble caractéristique des plaques séniles [44]. In vitro, l'isoforme E4 est particulièrement apte à promouvoir la polymérisation du peptide $A \beta$ en filaments amyloïdes [45]. Cette explication forme un tout cohérent avec l'hypothèse amyloï dergique. Il faut toutefois signaler que d'autres hypothèses pathogéniques on t été avancées qui sont fondées sur la liaison de l'ApoE à la protéine Tau, l'un des constituants des dégénérescences neuro-fibrillair es [46].

L'association de l'allèle E 4 avec d'autres types de démences fait actuellement l'objet de discussions intenses. L'association avec les démences vasculaires [47] ne pose pas de problèmes conceptuels particuliers si l'on considère d'une part que l'apoE4 est en soi un facteur de risque cardiovasculaire et que d'autre part de nombreuses démences vasculaires sont en fait des démences mixtes associant aux lésions ischémiques des plaques séniles et des dégénér escences neurofibrillaires. Il en va de même pour les démences à corps de Lewy dans lesquelles les lésions de la maladie d'Alzheimer sont souvent présentes conjointement [48]. L'implication de l'apoE4 dans une démence à prion, la maladie de Creutzfed-Jacob, a été suggérée [49] mais ce résultat est controversé [50]. Récemment, le rôle de l'allèle $\varepsilon 4$ dans le déclin cognitif de la personne agée non démente a également été envisagé [51]. Il est clair que nous abordons là une physiopathologie - celle du vieillissement cérébral - qui dépasse le cadre de la maladie d'Alzheimer. Une explication attrayante pour ce phénomène est le rôle joué par l'ApoE, par sa fonction de mobilisation du cholestérol, dans la maintenance et la réparation des membranes neuronales. Il semblerait que la vulnérabilité des neuron es vis-à-vis d'une agression dépende du génotype ApoE et que la présence de l'isoforme E4 constitue un facteur péjoratif lors de la réparation neuronale [52].

\section{Existe-t-il d'autres gènes candidats?}

Si l'on considère l'hypoth èse amyloïdergique, il est clair que diverses molécules présentes au sein des plaques pourraient jouer un rôle de chaperon similaire à l'ApoE. Le seuil de déclenchement de la maladie résulterait d'une balance entre les facteurs pro- et antiagrégants. Si cette hypothèse est exacte, nous devons trouver pour chacun des candidats potentiels des variants alléliques dont la fréquence chez les malades diffère de celle observée chez les témoins. Des travaux concernant l'un de ces candidats, l' $\alpha$ l antichymotrypsine (ACT), ont récemment été publiés [45, 53]. Il semblerait que le risque conféré par l'APOE $\varepsilon 4$ puisse être modifié par un polymorph isme situé au niveau de la séquence codant pour le peptide signal de l'ACT [53] mais ce résultat fait actuellement l'objet de critiques assez vives, l'essentiel de l'effet décrit venant d'une fluctuation de distribution dans l'un des groupes témoins. Pour notre part, nous nous sommes intéressés à un autre constituant des plaques, le peptide NAC (non amyloid component of senile plaques) [54] issu 


\section{RÉFÉRENCES}

45. Ma J, Yee A, Brewer HB, Das S, Potter H. Amyloid-associated proteins $\alpha 1$-antichymotrypsin and apolipoprotein E promote assembly of Alzheimer $\beta$-protein into filaments. Nature 1994 ; 372: 924

46. Strittmatter WI, Saunders AM, Goedert M, Weisgraber KH, Dong LM, Jakes R, Huang DY, Pericak-Vance M, Schmechel D, Roses A. Isoform specific interactions of apolipoprotein $\mathrm{E}$ with microtubule associated protein tau: implications for Alzheimer disease. Proc Natl Acad Sci USA 1994; 91 : $11183-6$.

47. Noguchi S, Murakami K Apolipoprotein $\mathbf{E}$ genotype and Alzheimer's disease. Lancet $1993 ; 342$ : 737.

48. Galasko D, Saitoh T, Xia Y, Thal LJ, Katzman R, Hill LR, Hansen L. The apolipoprotein $\mathrm{E}$ allele $\varepsilon 4$ is overrepresented in patients with the Lewy body variant of Alzheimer's disease. Neurology 1994; 44: 1950-1.

49. Amouyel P, Vidal O, Launay IM, Laplanche $\mathrm{JL}$. The apolipoprotein $\mathrm{E}$ alleles as major susceptibility factors for CreutzfedtJakob disease. Lancet 1994; 344: 1315-8.

50. Nakagawa $Y$, Kitamoto $T$, Furukawa $H$, Ogomori K, Tateishi J. Apolipoprotein $\mathrm{E}$ in Creutzfedt-Jakob disease. Lancet 1995; 345 : 68-9.

51. Reed T, Carmelli D, Swan GE, Breitner JCS, Welsh KA, Jarvik GP, Deeb S, Auwerx J. Lower cognitive performance in normal of der adult male twins carrying the apolipoprotein E 84 allele. Arch Neurol 1994; $1189-92$.

52. Nathan BP, Bellosta S, Sanan DA, Weisgraber KH, Mahley RW, Pitas RE. Differential effects of apolipoproteins $\mathrm{E} 3$ and $\mathrm{E} 4$ on neuronal growth in vitro. Science 1994; 264 : $850-2$.

53. Kamboh MI, Sanghera DK, Ferrel RE, Dekosky ST. ApoE 4 associated Alzheimer's disease risk is modified by $\alpha 1$-antichymotrypsin polymorphism. Nature Genet 1995; 10: $486-8$.

54. Campion D, Martin C, Heilig R, Charbonnier F, Moreau V, Flaman JM, Petit JL, Hannequin D, Brice A, Frebourg Th. The NACP/synuclein gene: chromosomal assignment and screening for alterations in $\mathrm{Alz}$ heimer disease. Genomics $1995 ; 26: 2547$.

55. ACMG/ASHG Working Group. Statement on use of apolipoprotein $E$ testing for Alzheimer disease. J Am Med Ass 1995; 274 : 1627-9.

56. Schellenberg GD. Genetic dissection of Alzheimer disease, a heterogeneous disorder. Proc Natl Acad Sci USA 1995; 92: 8552-9.

Pour des raisons de respect des normes éditoriales, de nombreuses réferences n'ont pu être citées. Les lecteurs intéressés poutront trontver ume bibliographie plus complète dans une rezue récente [56]. d'un précurseur présent dans les terminaisons synaptiques, la synucléine. Nous avons séquencé le gène de la synucléine chez 26 proposants correspondant à des formes familiales précoces. Nous n'avons pas retrouvé de polymorphisme dans la séquence codante du gène chez ces patients; toutefois nous avons pu mettre en évidence la présence d'un épissage alternatif, ce qui laisse ouverte la possibilité d'une dysrégulation dans l'équilibre des différents transcrits chez les malades.

\section{Conclusion}

Il y a dix ans, notre ignorance des causes de la maladie d'Alzheimer était complète et le rôle des facteurs génétiques controversé. Nous savons maintenant que certains cas familiaux précoces obéissent à un déterminisme autosomique dominant. Au sein de ces formes il existe une hétérogénéité étiologique. Plusieurs gènes distincts (APP, présénilines 1 et 2) sont impliqués dans cette sous-entité mendélienne. Pour les autres cas, le poids d'autres facteurs génétiques (APOE) est important. Cependant, dans ces cas le déterminisme n'est pas mendélien, et l'allèle $\mathrm{APOE} \varepsilon 4$ n'agit que comme facteur de risque en conjonction avec d'autres facteurs génétiques ou liés à l'environnement encore inconnus. Ces considérations sont importantes si l'on veut aborder la question du diagnostic prédictif de la MA. Il est clair que nous avons à présent les moyens de réaliser ce diagnostic chez des sujets asymptomatiques dans les rares familles où il existe une mutation des gènes $A P P$, préséniline 1 ou 2 . La mise en place effective de ce type de diagnostic pose des problèmes éthiques similaires à ceux déjà rencontrés dans la maladie de Huntington. Il nécessitera donc d'édicter une série de règles déjà appliquées pour cette dernière maladie: diagnostic possible uniquement chez des sujets majeurs, confidentialité, délai de réflexion après information initiale pour le demandeur, suivi psychologique. Pour ce qui concerne l'APOE, l'existence d'un risque relatif - imparfaitement mesuré actuellement - lié aux génotypes $\varepsilon 4 / \varepsilon 4$ et $\varepsilon 4 / X$ ne justifie pas, à notre sens, la généralisation des typages APOE. A l'étape actuelle, ceux-ci doivent rester limités aux protocoles de recherche, d'autant que nous ne disposons d'aucune thérapeutique préventive chez les sujets (quelques millions de personnes dans la population française) porteurs de ce facteur de risque. Ces conclusions sont également celles d'un groupe consensus constitué sous les auspices de la société américaine de génétique humaine [55]. Le principal intérêt des recherches actuelles est qu'elles ont permis de différencier des sousgroupes au sein de la MA. Chacun de ces sous-groupes étant déterminé par des mécanismes moléculaires précis, des traitements spécifiques seront certainement à envisager

\section{Summary}

Genetic determinism of Alzheimer's disease

The genetic determinism of $\mathrm{Alz}$ heimer's disease (AD) is heterogeneous. Autosomal dominant forms of the disease involve the amyloid precursor protein (APP) gene located on chromosome 21 , the presenilin-1 gene on chromosome 14 and the presenilin-2 gene on chromosome 1. Altogether these genes account for less than $20 \%$ of earlyonset $\mathrm{AD}$ cases (onset before 60). APP mutations cause $\mathrm{AD}$ by altering the APP processing in a way that is amyloidergic. How mutations of presenilin-1 and -2 act to promote $\mathrm{AD}$ neuropathology is currently unknown. Besides these major genes, the apolipoprotein $\mathrm{E}$ (APOE) gene, located on chromosome 19 , is also implicated in the diathesis of the disease. A strong association has been described between $\mathrm{AD}$ and the APOE $\varepsilon 4$ allele. This allele is a risk factor for AD whatever the sex and the age. Cases bearing the $\varepsilon 4$ allele have more deposition of amyloid $\beta$ peptide in brain than those who do not. APOEE 4 isof orm could act as a molecular chaperone conforming soluble $\beta$ peptide into an insoluble amyloid form.

\section{TIRÉS À PART}

D. Campion. 